\title{
Estimating the Reliability Function of Parallel Stress-Strength Model for the Generalized Inverted Kumaraswamy Distribution
}

\author{
Hakeeem Hussain Hamad Salih* and Nada S. Karam \\ Al-Mustanseriya University, College of Education, Mathematics Department, Baghdad, Iraq
}

\begin{tabular}{ll}
\hline \multicolumn{1}{c}{ Articles Information } & \multicolumn{1}{c}{ Abstract } \\
\hline Received: & This paper discusses reliability of the stress-strength model, the reliability \\
10.02 .2020 & functions $R_{1}$ and $R_{2}$ for two and three components which has two and three \\
Accepted: & strengths independently are exposed to a common stress respectively using \\
30.05 .2020 & Generalized inverted Kumaraswamy distribution(GIKD) with unknown shape \\
Published: & parameter and known shape and scale parameters, after that the parameters \\
26.09 .2020 & are estimate from a stress- strength model. Estimate the reliability $R_{1}, R_{2}$ by two \\
\hline Keywords: & methods (ranked set sampling and Bayes). A numerical simulation study a \\
Reliability function & comparison between the two estimators by Mean square error is performed. It is \\
Generalized inverted & found that best estimator between the two estimators is Bayes estimation \\
Kumaraswamy distribution & method. \\
Strength-stress model &
\end{tabular}

DOI: 10.22401/ANJS.23.3.09

${ }^{*}$ Corresponding author: hak88hak88@gmail.com

\section{Introduction}

The stress-strength model is an importance subject in reliability literature. In the statistical approach to the stress-strength model, the considerations depend on the assumption that the component strengths are independently and identically distributed and are subjected to a common stress [1].

Let $\mathrm{X}$ be a random variable refer to strength which follow Generalized inverted Kumaraswamy distribution (GIKD).

Let $\mathrm{y}$ is random variable refer to stress which follow Generalized inverted Kumaraswamy distribution (GIKD).

The system reliability using when $\mathrm{X}$ and $\mathrm{Y}$ are independent and identical.

Let $\mathrm{X}$ be a strength random variable subjected to a common stress $\mathrm{Y}$ then the reliability of the system contain one component is $R=P(X>Y)$. The reliability of a system is the probability that it is operating under stated environmental conditions [2].

The stress- strength reliability describes the life of a component which has random strength $\mathrm{X}$ that is subjected to a random stress $\mathrm{Y}$, when the stress applied to it exceeds its strength the component fails instantly and the component will function satisfactorily till $X>Y$ therefore $R=P(Y<X)$ is a measure of a component reliability. The stress-strength model is used in many applications in physics, in almost all areas of knowledge, especially in engineering [3].

\section{The Reliability Mathematical Formula}

In this model strength-stress random variables of components having strength $\left(X_{i ;} i=1,2,3\right)$ and exposed to stress $(Y)$, where $X_{i}$ and $Y$ are independently identically distributed Generalized inverted Kumaraswamy distribution (GIKD) random variables with common known shape and scale parameters $\alpha, \gamma$ respectively and unknown shape parameters $\beta_{\mathrm{i} ;} i=1,2,3$ and $\mathrm{b}$, such that $\mathrm{b}$ is shape parameter with stress random variables $Y$.

The system reliability $R 1$ of two parallel components having strength variables $X_{i} ; i=1,2$ are subjected to a common random stress $Y$, is the probability of maximum two strengths under one stress, then the system reliability $R_{1}$ is given by:[2].

$$
R_{1}=p\left[y<\max \left\{x_{1}, x_{2}\right\}\right]
$$

where $X_{1}, X_{2}, Y$ are mutually independently identically distributed random variables.

The system reliability, $R_{1}$, can be written as:

$$
\begin{aligned}
R_{1} & =1-p\left[y>\max \left\{x_{1}, x_{2}\right\}\right] \\
& =1-\int_{0}^{\infty} \int_{0}^{y} \int_{0}^{y} f\left(x_{1}, x_{2}, y\right) d x_{1} d x_{2} d y \\
& =1-\int_{0}^{\infty} \int_{0}^{y} f\left(x_{1}\right) d x_{1} \int_{0}^{y} f\left(x_{2}\right) d x_{2} f(y) d y \\
R_{1} & =1-\int_{0}^{\infty} F x_{1}(y) F x_{2}(y) f(y) d y
\end{aligned}
$$

Following the same paradigm, we have proposed a formula to find the reliability $R_{2}$ in case three parallel components having three independent strengths $X_{1}, X_{2} X_{3}$ and exposed to common stress $Y$ and which is the probability of the maximum of the three strengths under one stress, then the system reliability $R_{2}$ is given by: 


\section{Al-Nahrain Journal of Science}

ANJS, Vol.23 (3), September, 2020, pp. $68-75$

$$
\begin{aligned}
R_{2} & =p\left[y<\max \left\{x_{1}, x_{2}, x_{3}\right\}\right] \\
& =1-p\left[y>\max \left\{x_{1}, x_{2}, x_{3}\right\}\right] \\
& =1-\int_{0}^{\infty} \int_{0}^{y} \int_{0}^{y} \int_{0}^{y} f\left(x_{1}, x_{2}, x_{3}, y\right) d x_{1} d x_{2} d x_{3} d y \\
& =1-\int_{0}^{\infty} \int_{0}^{y} f\left(x_{1}\right) d x_{1} \int_{0}^{y} f\left(x_{2}\right) d x_{2} \int_{0}^{y} f\left(x_{3}\right) d x_{3} f(y) d y \\
R_{2} & =1-\int_{0}^{\infty} F x_{1}(y) F x_{2}(y) F x_{3}(y) f(y) d y
\end{aligned}
$$

\section{Generalized Inverted Kumaraswamy Distribution (GIKD)}

Kumaraswamy [5] obtained a distribution, which is derived from beta distribution after fixing some parameters in beta distribution. The distribution is appropriate to natural phenomena whose outcomes are bounded from both sides, such as the individual's heights, test scores, temperatures and hydrological daily data of rain fall.

The probability density function and distribution function of the Kumaraswamy distribution are given, respectively, by:

$$
\begin{aligned}
f(x ; \alpha, \beta)= & \left.\alpha \beta x^{\alpha-1}\left[1-x^{\alpha}\right)\right]^{\beta-1}, \alpha, \beta>0, x \in \\
& (0,1) \\
F(x ; \alpha, \beta)= & \left.1-\left[1-x^{\alpha}\right)\right]^{\beta}
\end{aligned}
$$

The inverted distribution have many applications in different fields such as; biological sciences, life testing problems, engineering sciences, environmental studies and econometrics. In the last two decades, the researchers proposed many inverted distributions due to its great applications; studied and [6] investigated the inverted Kumaraswamy distribution.

Abd Al-Fattah et al. [6] derived the inverted Kumaraswamy distribution from Kumaraswamy (Kum) distribution using the transformation $t=X^{-1}-1$. When $X \sim \operatorname{Kum}(\alpha, \beta)$ where $\alpha$ and $\beta$ are shape parameters, then probability density function and distribution function of the inverted Kumaraswamy distribution are given, respectively, by:

$$
\begin{aligned}
f(t, \alpha, \beta)= & \alpha \beta(1+t)^{-(\alpha+1)}\left[1-(1+t)^{-\alpha}\right]^{\beta-1}, \\
& x>0 \\
F(t, \alpha, \beta)= & {\left[1-(1+t)^{-\alpha}\right]^{\beta} }
\end{aligned}
$$

where $\alpha>0$ and $\beta>0$ are two shape parameters.

Iqbal [7] introduced an extension of the inverted Kumaraswamy distribution called the generalized inverted Kumaraswamy distribution (GIKD) by using transformation $t=x^{\gamma}$ with has probability density function and distribution function are given, respectively, by:

Let $\mathrm{X}$ be a random variable refer to strength then if $X \sim G I K(\alpha, \beta, \gamma)$, then the pdf of GIKD is [6], [7]:

$$
f(x ; \alpha, \beta, \gamma)=\alpha \beta \gamma x^{\gamma-1}\left(1+x^{\gamma}\right)^{-(1+\alpha)}[1-(1+
$$

where $\alpha, \beta, \gamma>0, x>0, \alpha, \beta$ are shape parameters.

The CDF of GIKD is:

$$
F(x ; \alpha, \beta, \gamma)=\left[1-\left(1+x^{\gamma}\right)^{-\alpha}\right]^{\beta}
$$

Let $\mathrm{y}$ is random variable refer to stress then $Y \sim G I K(\alpha, b, \gamma)$. Also the pdf of GIKD is:

$$
f(y ; \alpha, b, \gamma)=\alpha b \gamma y^{\gamma-1}\left(1+y^{\gamma}\right)^{-(1+\alpha)}[1-(1+
$$

such that $y>0, \alpha, b, \gamma>0$ and the cdf of

GIKD is:

$$
F(y ; \alpha, b, \gamma)=\left[\left(1-\left(1+y^{\gamma}\right)^{-\alpha}\right]^{b}\right.
$$

3.1 Two Strength-one stress component reliability Let $X_{i}$ be the strength $\sim G I K\left(\alpha, \beta_{i}, \gamma\right), i=1,2$ and $Y$ is stress $\sim G I K(\alpha, b, \gamma)$

$$
F\left(x_{i} ; \alpha, \beta_{i}, \gamma\right)=\left[\left(1-\left(1+x_{i}^{\gamma}\right)^{-\alpha}\right]^{\beta_{i}}, i=1,2\right.
$$

Substitute (5) and (6) in (1)

$$
\begin{aligned}
R_{1}= & 1-\int_{0}^{\infty} F x_{1}(y) F x_{2}(y) f(y) d y \\
= & 1-\int_{0}^{\infty}\left[\left(1-\left(1+y^{\gamma}\right)^{-\alpha}\right]^{\beta_{1}}[(1-(1+\right. \\
& \left.\left.y^{\gamma}\right)^{-\alpha}\right]^{\beta_{2}} \alpha b \gamma y^{\gamma-1}\left(1+y^{\gamma}\right)^{-(1+\alpha)}[1-(1+ \\
& \left.\left.y^{\gamma}\right)^{-\alpha}\right]^{b-1} d y \\
R_{1}= & 1-b \int_{0}^{\infty}\left[1-\left(1+y^{\gamma}\right)^{-\alpha}\right]^{\beta_{1}+\beta_{2}+b-1} \alpha(1+ \\
& \left.y^{\gamma}\right)^{-(1+\alpha)} \gamma y^{\gamma-1} d y
\end{aligned}
$$

By using:

$$
\int_{0}^{\infty}[f(y)]^{n} \frac{d[f(y)]}{d y} d y=\left.\frac{[f(y)]^{n+1}}{n+1}\right|_{0} ^{\infty}
$$

where $\frac{d[f(y)]}{d y}$ is the derivative of $f(y)$.

In the same way $\alpha\left(1+y^{\gamma}\right)^{-(1+\alpha)} \gamma y^{\gamma-1}$ is the derivative of $1-\left(1+y^{\gamma}\right)^{-\alpha}$

$$
\begin{aligned}
& R_{1}=1-\left.\left\{\frac{b\left[\left(1-\left(1+y^{\gamma}\right)^{-\alpha}\right]^{\beta_{1}+\beta_{2}+b}\right.}{\beta_{1}+\beta_{2}+b}\right\}\right|_{0} ^{\infty} \\
& R_{1}=1-\frac{b}{\beta_{1}+\beta_{2}+b}
\end{aligned}
$$

\subsection{Three strength-one stress component reliability}

If $x_{i}$ is strength $\sim \operatorname{GIK}\left(\alpha, \beta_{i}, \gamma\right), i=1,2,3$ and $Y$ is stress $\sim \operatorname{GIK}(\alpha, b, \gamma)$

$$
F\left(x_{i} ; \alpha, \beta, \gamma\right)=\left[\left(1-\left(1+x_{i}^{\gamma}\right)^{-\alpha}\right]^{\beta_{i}}, i=1,2,3\right.
$$

Substitute (5) and (8) in (2)

$$
\begin{aligned}
R_{2}= & 1-\int_{0}^{\infty} F x_{1}(y) F x_{2}(y) F x_{3}(y) f(y) d y \\
= & 1-\int_{0}^{\infty}\left[\left(1-\left(1+y^{\gamma}\right)^{-\alpha}\right]^{\beta_{1}}[(1-(1+\right. \\
& \left.\left.y^{\gamma}\right)^{-\alpha}\right]^{\beta_{2}}\left[\left(1-\left(1+y^{\gamma}\right)^{-\alpha}\right]^{\beta_{3}} \alpha b \gamma y^{\gamma-1}(1+\right. \\
& \left.y^{\gamma}\right)^{-(1+\alpha)}\left[1-\left(1+y^{\gamma}\right)^{-\alpha}\right]^{b-1} d y \\
= & 1-b \int_{0}^{\infty}\left[\left(1-\left(1+y^{\gamma}\right)^{-\alpha}\right]^{\beta_{1}+\beta_{2}+\beta_{3}+b-1} \alpha(1+\right. \\
& \left.y^{\gamma}\right)^{-(1+\alpha)} \gamma y^{\gamma-1} d y
\end{aligned}
$$

By using $\int_{0}^{\infty}[f(y)]^{n} \frac{d[f(y)]}{d y} d y=\left.\frac{[f(y)]^{n+1}}{n+1}\right|_{0} ^{\infty}$, where $\frac{d[f(y)]}{d y}$ is the derivative of $f(y)$.

In the same way $\alpha\left(1+y^{\gamma}\right)^{-(1+\alpha)} \gamma y^{\gamma-1}$ is derivative to:

$$
\begin{aligned}
& 1-\left(1+y^{\gamma}\right)^{-\alpha}=1-\left.\left\{\frac{b\left[\left(1-\left(1+y^{\gamma}\right)^{-\alpha}\right]^{\beta_{1}+\beta_{2}+\beta_{3}+b}\right.}{\beta_{1}+\beta_{2}+\beta_{3}+b}\right\}\right|_{0} ^{\infty} \\
& R_{2}=1-\frac{b}{\beta_{1}+\beta_{2}+\beta_{3}+b}
\end{aligned}
$$

4. Methods of Estimating the Reliability Function In this sub section, we will estimate the unknown shape parameters $\beta, \beta_{1}, \beta_{2}, \beta_{3}, b$ of GIKD and estimate the system reliability functions $R_{1}, R_{2}$ have been estimated by two different methods of estimation; Ranked set sampling method and Bayes estimation method. 


\section{Al-Nahrain Journal of Science}

ANJS, Vol.23 (3), September, 2020, pp. $68-75$

\subsection{Ranked set sampling method (RSS)}

McIntyre introduced the Ranked set sampling for improved the efficiency of the sample mean as an estimator of the population mean in situations when the characteristic of interest was difficult or expensive to measure [8] and [9].

Assume that we have random sample $X_{1}, X_{2}, \ldots, X_{n}$ for GIKD. Let $X_{(1)}, X_{(2)} \ldots, X_{(n)}$ be an order statistic, the pdf of $X_{(i)}$ is:

$$
g_{\left(X_{i}\right)}=\frac{n !}{(i-1) !(n-i) !}\left[F\left(x_{i}\right)\right]^{i-1}\left[1-F\left(x_{i}\right)\right]^{n-i} f\left(x_{i}\right)
$$

By substituting (3), (4) in (10)

$$
g_{\left(X_{i}\right)}=\frac{n !}{(i-1) !(n-i) !}\left[\left[1-\left(1+x^{\gamma}\right)^{-\alpha}\right]^{\beta}\right]^{i-1}\left[1-\left[1-\left(1+x^{\gamma}\right)^{-\alpha}\right]^{\beta}\right]^{n-i} \alpha \beta \gamma x^{\gamma-1}\left(1+x^{\gamma}\right)^{-(1+\alpha)}\left[1-\left(1+x^{\gamma}\right)^{-\alpha}\right]^{\beta-1}
$$

The likelihood function of $g_{\left(X_{i}\right)}$ is

$$
\begin{gathered}
L=\left[\frac{n !}{(i-1) !(n-i) !}\right]^{n} \alpha^{n} \beta^{n} \gamma^{n} \prod_{i=1}^{n}\left[\left[1-\left(1+x_{i}^{\gamma}\right)^{-\alpha}\right]^{\beta}\right]^{i-1} \prod_{i=1}^{n}\left[1-\left[1-\left(1+x_{i}^{\gamma}\right)^{-\alpha}\right]^{\beta}\right]^{n-i} \prod_{i=1}^{n} x_{i}^{\gamma-1} \prod_{i=1}^{n}(1+ \\
\left.x_{i}^{\gamma}\right)^{-(1+\alpha)} \prod_{i=1}^{n}\left[1-\left(1+x_{i}^{\gamma}\right)^{-\alpha}\right]^{\beta-1}
\end{gathered}
$$

The logarithm to relation (11) is:

$$
\begin{aligned}
\operatorname{Lnl}= & n \ln \left[\frac{n !}{(i-1) !(n-i) !}\right]+n \ln \alpha+n \ln \beta+n \ln \gamma+\sum_{i=1}^{n} \beta(i-1) \ln \left[1-\left(1+x_{i}^{\gamma}\right)^{-\alpha}\right]+\sum_{i=1}^{n}(n-i) \ln [1-[1-(1+ \\
& \left.\left.\left.x_{i}^{\gamma}\right)^{-\alpha}\right]^{\beta}\right]+(\gamma-1) \sum_{i=1}^{n} \ln x_{i}-(1+\alpha) \sum_{i=1}^{n} \ln \left(1+x_{i}^{\gamma}\right)+(\beta-1) \sum_{i=1}^{n} \ln \left[1-\left(1+x_{i}^{\gamma}\right)^{-\alpha}\right]
\end{aligned}
$$

We want in this paper to estimate $\beta$, so taking partial derivatives to equation (12) with respect to $\beta$ and equal to zero, such that $\beta$ is unknown shape parameter, to get after that the reliability, we get:

$$
\begin{aligned}
& \frac{d L n l}{d \beta}=\frac{n}{\beta}+\sum_{i=1}^{n}(i-1) \ln \left[\left(1-\left(1+x_{i}^{\gamma}\right)^{-\alpha}\right]-\sum_{i=1}^{n}(n-i) \frac{\left[( 1 - ( 1 + x _ { i } ) ^ { - \alpha } ] ^ { \beta } \operatorname { l n } \left[\left(1-\left(1+x_{i}\right)^{-\alpha}\right]\right.\right.}{1-\left[\left(1-\left(1+x_{i}\right)^{-\alpha}\right]^{\beta}\right.}+\sum_{i=1}^{n} \ln \left[1-\left(1+x_{i}^{\gamma}\right)^{-\alpha}\right]=0\right. \\
& \hat{\beta}_{R S S}=\frac{-n}{\sum_{i=1}^{n}(i-1) \ln \left[\left(1-\left(1+x_{i}{ }^{\gamma}\right)^{-\alpha}\right]-\sum_{i=1}^{n}(n-i) \frac{\left[( 1 - ( 1 + x _ { i } ) ^ { - \alpha } ] ^ { \beta } \operatorname { l n } \left[\left(1-\left(1+x_{i}^{\gamma}\right)^{-\alpha}\right]\right.\right.}{1-\left[\left(1-\left(1+x_{i}^{\gamma}\right)^{-\alpha} \beta_{0}\right.\right.}+\sum_{i=1}^{n} \ln \left[1-\left(1+x_{i}{ }^{\gamma}\right)^{-\alpha}\right]\right.}
\end{aligned}
$$

where $\beta_{o}$ is initial value.

To find $\hat{R}_{1 R S S}$ and $\hat{R}_{2 R S S}$, we must estimate $\mathrm{b}, \beta_{1}, \beta_{2}$ and $\beta_{3}$ since existing in relations. In the same way in case $\hat{\beta}_{R S S}$, we will find $\hat{b}_{R S S}$.

Let $\mathrm{Y}$ stress random sample has $\operatorname{GIK}(\alpha, b, \gamma)$ distribution with sample size $\mathrm{m}$ where $b$ is unknown parameter, the RSS estimator for $\left(\hat{b}_{R S S}\right)$, can be derived as:

$$
\hat{b}_{R S S}=\frac{-m}{\sum_{j=1}^{m}(j-1) \ln \left[\left(1-\left(1+y_{j} \gamma^{-\alpha}\right]-\sum_{j=1}^{m}(m-j) \frac{\left[( 1 - ( 1 + y _ { j } ^ { \gamma } ) ^ { - \alpha } ] ^ { b } \operatorname { l n } \left[\left(1-\left(1+y_{j}^{\gamma}\right)^{-\alpha}\right]\right.\right.}{1-\left[\left(1-\left(1+y_{j}\right)^{-\alpha}\right]^{b}\right.}+\sum_{j=1}^{m} \ln \left[1-\left(1+y_{j} \gamma^{-\alpha}\right]\right.\right.\right.}
$$

where $b_{o}$ is initial value.

Let $X_{1_{i_{1}}} ; i_{1}=1,2, \ldots, n_{1}, X_{2_{i_{2}}} ; i_{2}=1,2, \ldots, n_{2}$ and $X_{3_{i_{3}}} ; i_{3}=1,2, \ldots, n_{3}$ are strengths random sample which has $\operatorname{GIK}\left(\alpha, \beta_{1}, \gamma\right), \operatorname{GIK}\left(\alpha, \beta_{2}, \gamma\right), \operatorname{GIK}\left(\alpha, \beta_{3}, \gamma\right)$ distributions, with sample size $n_{1}, n_{2}$ and $n_{3}$, respectively, where $\beta_{1}, \beta_{2}$ and $\beta_{3}$ are unknown parameters and by using the same manner,the RSS estimators to the unknown parameters $\beta_{1}, \beta_{2}$ and $\beta_{3}$ are:

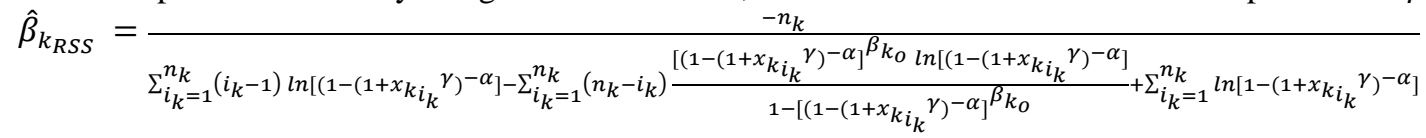

where $k=1,2,3$.

By substituting (13) and (14) into relations (7) and (9), respectively, we get the reliability estimators $\hat{R}_{1 \text { RSS }}$ and $\hat{R}_{2 R S S}$

$$
\begin{aligned}
& \hat{R}_{1 R S S}=\frac{\hat{b}_{R S S}}{\hat{\beta}_{1 R S S}+\widehat{\beta}_{2} R S S+\hat{b}_{R S S}} \\
& \hat{R}_{2 R S S}=\frac{\hat{b}_{R S S}}{\widehat{\beta}_{1 R S S}+\widehat{\beta}_{2} R S S+\widehat{\beta}_{3 R S S}+\hat{b}_{R S S}}
\end{aligned}
$$

\subsection{Bayes estimation methods}

We have to find the Bayes estimator for $\beta$ using non informative prior distribution $f(\beta)$ based on modified extension of Jeffery Prior and square error loss function [10] and [11].

To get the prior distribution to the parameter $\beta$ using the modified extension of Jeffery, prior can be found by:

$$
f(\beta) \propto\left(-n E\left[\frac{d^{2} \ln f}{d \beta^{2}}\right]\right)^{c}
$$

To find $\left(-n E\left[\frac{d^{2} l n f}{d \beta^{2}}\right]\right)$ take natural logarithm to pdf in relation (3)

$$
\begin{aligned}
\operatorname{Ln} f= & \ln \alpha+\ln \beta+\ln \gamma+(\gamma-1) \ln x- \\
& (1+\alpha) \ln \left(1+x^{\gamma}\right)+(\beta-1) \ln [1-(1+ \\
& \left.\left.x^{\gamma}\right)^{-\alpha}\right]
\end{aligned}
$$

Taking partial derivative with respect to $\beta$

$$
\frac{d \ln f}{d \beta}=\frac{1}{\beta}+\ln \left[1-\left(1+x^{\gamma}\right)^{-\alpha}\right]
$$

Again taking second partial derivative with respect to $\beta$, we get: 


\section{Al-Nahrain Journal of Science}

ANJS, Vol.23 (3), September, 2020, pp. $68-75$

$$
\begin{aligned}
& \frac{d^{2} \ln f}{d \beta^{2}}=\frac{-1}{\beta^{2}} \\
& \begin{aligned}
E\left(\frac{d^{2} \ln f}{d \beta^{2}}\right) & =E\left(\frac{-1}{\beta^{2}}\right) \\
& =\frac{-1}{\beta^{2}}-n E\left(\frac{d^{2} \ln f}{d \beta^{2}}\right) \\
& =\frac{n}{\beta^{2}}
\end{aligned}
\end{aligned}
$$

Substitute (18) in (17), we get $f(\beta) \propto\left(\frac{n}{\beta^{2}}\right)^{c}$

$$
f(\beta)=k n^{c} \beta^{-2 c}
$$

which is the prior distribution.

The likelihood function to pdf in relation (3) is:

$L\left(x_{i}, \alpha, \beta, \gamma\right)=\alpha^{n} \beta^{n} \gamma^{n} \prod_{i=1}^{n} x_{i}^{\gamma-1} \prod_{i=1}^{n}\left(1+x_{i}{ }^{\gamma}\right)^{-(1+\alpha)} \prod_{i=1}^{n}\left[1-\left(1+x_{i}^{\gamma}\right)^{-\alpha}\right]^{\beta-1}$

let $L\left(x_{i}, \alpha, \beta, \gamma\right)=L$, then the posterior distribution is:

$$
f\left(\beta / x_{1}, x_{2}, \ldots, x_{n}\right)=\frac{L f(\beta)}{\int L f(\beta) d \beta}
$$

Substitute (19) and (20) in (21), we get:

$$
\begin{aligned}
& f\left(\beta / x_{1}, x_{2}, \ldots, x_{n}\right)=\frac{\alpha^{n} \beta^{n} \gamma^{n} \prod_{i=1}^{n} x_{i}^{\gamma-1} \Pi_{i=1}^{n}\left(1+x_{i}^{\gamma}\right)^{-(1+\alpha)} \prod_{i=1}^{n}\left[1-\left(1+x_{i}^{\gamma}\right)^{-\alpha} \beta \beta^{\beta-1} k n^{c} \beta^{-2 c}\right.}{\int \alpha^{n} \beta^{n} \gamma^{n} \prod_{i=1}^{n} x_{i}^{\gamma-1} \prod_{i=1}^{n}\left(1+x_{i}\right)^{-(1+\alpha)} \prod_{i=1}^{n}\left[1-\left(1+x_{i} \gamma\right)^{-\alpha}\right]^{\beta-1} k n^{c} \beta^{-2 c} d \beta} \\
& f\left(\beta / x_{1}, x_{2}, \ldots, x_{n}\right)=\frac{\beta^{n-2 c} \prod_{i=1}^{n}\left[1-(1+x \gamma)^{-\alpha}\right]^{\beta-1}}{\int \beta^{n-2 c} \prod_{i=1}^{n}\left[1-(1+x \gamma)^{-\alpha}\right]^{\beta-1} d \beta}
\end{aligned}
$$

To simplify (22), we take $\prod_{i=1}^{n}\left[1-\left(1+x_{i}^{\gamma}\right)^{-\alpha}\right]^{\beta-1}$

$$
\begin{gathered}
\prod_{i=1}^{n}\left[1-\left(1+x_{i}^{\gamma}\right)^{-\alpha}\right]^{\beta-1}=e^{\sum_{i=1}^{n} \ln \left[1-\left(1+x_{i}{ }^{\gamma}\right)^{-\alpha}\right]^{\beta-1}}=e^{\beta \sum_{i=1}^{n} \ln \left[1-\left(1+x_{i} \gamma\right)^{-\alpha}\right]} e^{-\sum_{i=1}^{n} \ln \left[1-\left(1+x_{i} \gamma\right)^{-\alpha}\right]} \\
\prod_{i=1}^{n}\left[1-\left(1+x_{i}^{\gamma}\right)^{-\alpha}\right]^{\beta-1}=e^{-\sum_{i=1}^{n} \beta \ln \left[1-\left(1+x_{i} \gamma\right)^{-\alpha}\right]^{-1}} e^{-\sum_{i=1}^{n} \ln \left[1-\left(1+x_{i}\right)^{-\alpha}\right]}
\end{gathered}
$$

by using the rule $a^{x}=e^{\ln a^{x}}=e^{x \ln a}=e^{-x \ln a^{-1}}$. Substitute (23) in (22), we get:

$$
f\left(\beta / x_{1}, x_{2}, \ldots, x_{n}\right)=\frac{\left.\left.\beta^{n-2 c} e^{-\sum_{i=1}^{n} \beta \ln [1-(1+x} \gamma\right)^{-\alpha}\right]^{-1} e^{-\sum_{i=1}^{n} 1 \ln \left[1-(1+x \gamma)^{-\alpha}\right]}}{\left.\left.\int \beta^{n-2 c} e^{-\sum_{i=1}^{n} \beta \ln [1-(1+x} \gamma\right)^{-\alpha}\right]^{-1} e^{-\sum_{i=1}^{n} 1 \ln \left[1-(1+x \gamma)^{-\alpha}\right]}} d \beta
$$

Since the integral to $\beta$ and similar the $\frac{e^{-\sum_{i=1}^{n} 1 \ln \left[1-(1+x \gamma)^{-\alpha}\right]}}{e^{-\sum_{i=1}^{n} 1 \ln \left[1-(1+x \gamma)^{-\alpha}\right]}}$, so we get:

$$
f\left(\beta / x_{1}, x_{2}, \ldots, x_{n}\right)=\frac{\beta^{n-2 c} e^{-\sum_{i=1}^{n} \beta \ln \left[1-\left(1+x_{i} \gamma\right)^{-\alpha}\right]^{-1}}}{\int \beta^{n-2 c} e^{-\sum_{i=1}^{n} \beta \ln \left[1-\left(1+x_{i} \gamma\right)^{-\alpha}\right]^{-1} d \beta}}=\frac{\beta^{n-2 c} e^{-\sum_{i=1}^{n} \beta \ln \left[1-\left(1+x_{i} \gamma\right)^{-\alpha}\right]^{-1}}}{\frac{\Gamma(n-2 c+1)}{\left(\sum_{i=1}^{n} \ln \left[1-\left(1+x_{i} \gamma\right)^{-\alpha}\right]^{-1}\right)^{n-2 c+1}}}
$$

We can find $\hat{\beta}_{\text {bays }}$ by using the loss function. The square loss function which is defined as bellow:

$$
L(\hat{\beta}, \beta)=(\hat{\beta}-\beta)^{2}
$$

then the risk function $R(\hat{\beta}, \beta)$ appears as:

$$
\begin{aligned}
R(\hat{\beta}, \beta) & =E[\mathrm{~L}(\hat{\beta}, \beta)] \\
& =E\left[(\hat{\beta}-\beta)^{2}\right] \\
& =\int_{0}^{\infty}(\hat{\beta}-\beta)^{2} f\left(\beta / x_{1}, x_{2}, \ldots, x_{n}\right) \mathrm{d} \beta
\end{aligned}
$$

By substitute equation (24) in (25), we get:

$$
\begin{aligned}
& R(\hat{\beta}, \beta)=\int_{0}^{\infty}\left(\hat{\beta}^{2}-2 \hat{\beta} \beta+\beta^{2}\right) \frac{\beta^{n-2 c} e^{-\sum_{i=1}^{n} \beta \ln \left[1-\left(1+x_{i}{ }^{\gamma}\right)^{-\alpha}\right]^{-1}}}{\left(\sum_{i=1}^{n} \ln \left[1-\left(1+x_{i}\right)^{-\alpha}\right]^{-1}\right)^{n-2 c+1}} \mathrm{~d} \beta \\
& =\int_{0}^{\infty}\left(\hat{\beta}^{2} \frac{\beta^{n-2 c} e^{-\sum_{i=1}^{n} \beta \ln \left[1-\left(1+x_{i} \gamma\right)^{-\alpha}\right]^{-1}}}{\Gamma(n-2 c+1)}-2 \hat{\beta} \frac{\beta^{n-2 c+1} e^{-\sum_{i=1}^{n} \beta \ln \left[1-\left(1+x_{i}^{\gamma}\right)^{-\alpha}\right]^{-1}}}{\frac{\left.\sum_{i=1}^{n} \ln \left[1-\left(1+x_{i} \gamma\right)^{-\alpha}\right]^{-1}\right)^{n-2 c+1}}{\left(\sum_{i=1}^{n} \ln \left[1-\left(1+x_{i} \gamma\right)^{-\alpha}\right]^{-1}\right)^{n-2 c+1}}}+\frac{\beta^{n-2 c+2} e^{-\sum_{i=1}^{n} \beta \ln \left[1-\left(1+x_{i} \gamma\right)^{-\alpha}\right]^{-1}}}{\frac{\Gamma(n-2 c+1)}{\left(\sum_{i=1}^{n} \ln \left[1-\left(1+x_{i} \gamma\right)^{-\alpha}\right]^{-1}\right)^{n-2 c+1}}}\right) d \beta
\end{aligned}
$$

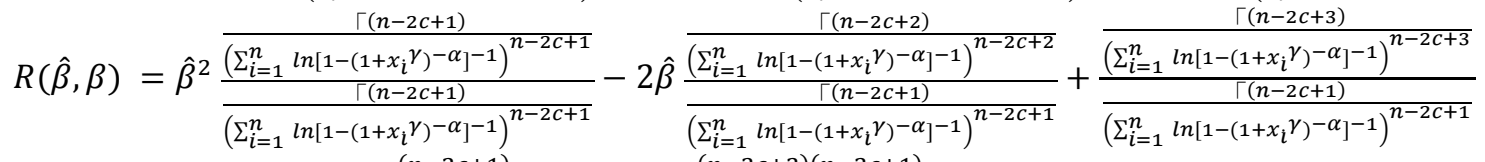

$$
\begin{aligned}
& \mathrm{R}(\hat{\beta}, \beta)=\hat{\beta}^{2}-2 \hat{\beta} \frac{(n-2 c+1)}{\left(\sum_{i=1}^{n} \ln \left[1-\left(1+x_{i}\right)^{-\alpha}\right]^{-1}\right)}+\frac{(n-2 c+2)(n-2 c+1)}{\left(\sum_{i=1}^{n} \ln \left[1-\left(1+x_{i}^{\gamma}\right)^{-\alpha}\right]^{-1}\right)^{2}}
\end{aligned}
$$

Taking partial derivatives with respect to $\hat{\beta}$ and equating the results to the zero:

$$
\frac{d R}{d \widehat{\beta}}=2 \hat{\beta}-2 \frac{(n-2 c+1)}{\left(\sum_{i=1}^{n} \ln \left[1-\left(1+x_{i} \gamma\right)^{-\alpha}\right]^{-1}\right)}=0
$$




\section{Al-Nahrain Journal of Science}

ANJS, Vol.23 (3), September, 2020, pp. $68-75$

Then the Bayes estimator is as bellow:

$$
\hat{\beta}_{\text {bays }}=\frac{n-2 c+1}{\sum_{i=1}^{n} \ln \left[1-\left(1+x_{i}\right)^{-\alpha}\right]^{-1}}
$$

In the same way, let $Y$ stress random sample has $\operatorname{GIK}(\alpha, b, \gamma)$ distribution with sample size $\mathrm{m}$ where $b$ is unknown parameter, the Bays estimator for $\left(\hat{b}_{\text {bays }}\right)$, can be derived as:

$$
\hat{b}_{\text {bays }}=\frac{m-2 c+1}{\sum_{j=1}^{m} \ln \left[1-\left(1+y_{j}\right)^{-\alpha}\right]^{-1}}
$$

Let $X_{1_{i_{1}}} ; i_{1}=1,2, \ldots, n_{1}, X_{2_{i_{2}}} ; i_{2}=1,2, \ldots, n_{2}$ and $X_{3_{i_{3}}} ; i_{3}=1,2, \ldots, n_{3}$ are strengths random sample has $\operatorname{GIK}\left(\alpha, \beta_{1}, \gamma\right)$, $\operatorname{GIK}\left(\alpha, \beta_{2}, \gamma\right), \operatorname{GIK}\left(\alpha, \beta_{3}, \gamma\right)$ distributions, with sample size $n_{1}, n_{2}$ and $n_{3}$, respectively, where $\beta_{1}, \beta_{2}$ and $\beta_{3}$ are unknown parameters. By using the same manner, the Bayes estimators to the unknown parameters $\beta_{1}, \beta_{2}$ and $\beta_{3}$ are:

$$
\hat{\beta}_{k_{\text {bays }}}=\frac{n_{k}-2 c+1}{\sum_{i_{k}=1}^{n_{k} \ln \left[1-\left(1+x_{k_{i}}{ }^{\gamma}\right)^{-\alpha}\right]^{-1}}}
$$

such that $k=1,2,3$, and $c$ is a constant.

By substitute the equations (26) and (27) in relations (7) and (9), respectively, we get the reliability estimators $\hat{R}_{1 \text { bays }}$ and $\hat{R}_{2 \text { bays }}$

$$
\begin{aligned}
& \hat{R}_{1 \text { bays }}=\frac{\hat{b}_{\text {bays }}}{\widehat{\beta}_{1 \text { bays }}+\widehat{\beta}_{2 \text { bays }}+\hat{b}_{\text {bays }}} \\
& \hat{R}_{2 \text { bays }}=\frac{\hat{\bar{b}}_{\text {bays }}}{\hat{\beta}_{1 \text { bays }}+\widehat{\beta}_{2} \text { bays }+\widehat{\beta}_{3 \text { bays }}+\hat{b}_{\text {bays }}}
\end{aligned}
$$

\section{Simulation}

A simulation study is a technique used to estimate the shape parameter of GIK, then utilizing shape parameter to estimate the reliability function through to two strengthone stress component and three strength-one stress component and comparing the results by utilizing mean squares error technique.

Generating Random Variables: Assume that $U$ be a random variable with the Uniform distribution in $(0,1)$,the samples random data are generated to follow Generalized Inverted Kumaraswamy distribution using the cdf of Generalized Inverted Kumaraswamy distribution and find the inverse of the distribution function as follow $U=$ $F(X) \rightarrow X=F^{-1}(U)$ by using equation (4), which is:

$$
\begin{aligned}
& \mathrm{F}(\mathrm{x} ; \alpha, \beta, \gamma)=\left[1-\left(1+\mathrm{x}^{\gamma}\right)^{-\alpha}\right]^{\beta} \\
& (\mathrm{F})^{\frac{1}{\beta}}=1-\left(1+\mathrm{x}^{\gamma}\right)^{-\alpha} \\
& \left(1+\mathrm{x}^{\gamma}\right)^{-\alpha}=1-(\mathrm{F})^{\frac{1}{\beta}} \\
& 1+\mathrm{x}^{\gamma}=\left[1-(\mathrm{F})^{\frac{1}{\beta}}\right]^{\frac{-1}{\alpha}} \\
& \mathrm{x}^{\gamma}=\left[1-(\mathrm{F})^{\frac{1}{\beta}}\right]^{\frac{-1}{\alpha}}-1 \\
& \mathrm{x}=\left[\left[1-(\mathrm{F}(\mathrm{x} ; \alpha, \beta, \gamma))^{\frac{1}{\beta}}\right]^{\frac{-1}{\alpha}}-1\right]^{\frac{1}{\gamma}}
\end{aligned}
$$

by letting $U=F(\mathrm{x} ; \alpha, \beta, \gamma)$, where $U$ is a Uniform continuous random variable defined on the interval $(0,1)$

$$
\mathrm{x}=\left[\left[1-(\mathrm{U})^{\frac{1}{\beta}}\right]^{\frac{-1}{\alpha}}-1\right]^{\frac{1}{\gamma}}
$$

We can use the generate data in technique (30) to get the random samples of the generalized inverted Kumaraswamy.

The simulation process has been designed in basic stages, which are important and necessary to find the estimation reliability of the shape parameter for the generalized Inverted Kumaraswamy distribution.

Step 1: Different sizes of the samples has been selected which are proportional with the effect of the sample size on the accuracy of the results obtained by using the two approaches of this paper, where the small sample size is choosing to be (15), medium sample size to be (30) and the large sample size to be (90).

The random samples $y_{1}, y_{2}, \ldots, y_{m}, x_{11}, x_{12}, \ldots, x_{1 n 1}, x_{21}$, $x_{22}, \ldots, x_{2 n 2}, x_{31}, x_{32}, \ldots, x_{3 n 3}$, of sizes $\left(m, n_{1}, n_{2}, n_{3}\right)$, such that the sample sizes are $(15,15,15,15),(30,30,30,30)$, $(90,90,90,90),(15,30,30,90), \quad(30,90,90,30) \quad$ and $(15,30,90,15)$.

Step 2: Real parameters values are selected for 6 experiments $\left(b, \beta_{1}, \beta_{2}, \beta_{3}, \alpha, \gamma\right)$ in the following table:

\begin{tabular}{c|l|l|l|l|l|l} 
Experiment & $\boldsymbol{b}$ & $\boldsymbol{\beta}_{\mathbf{1}}$ & $\boldsymbol{\beta}_{\mathbf{2}}$ & $\boldsymbol{\beta}_{\mathbf{3}}$ & $\boldsymbol{\alpha}$ & $\boldsymbol{\gamma}$ \\
\hline 1 & 2 & 1 & 3 & 1.5 & 1 & 1 \\
2 & 2 & 1 & 3 & 1.5 & 2 & 1 \\
3 & 2 & 1 & 3 & 1.5 & 1 & 2 \\
4 & 3 & 2.5 & 3.1 & 2.8 & 1 & 1 \\
5 & 3 & 2.5 & 3.1 & 2.8 & 2 & 1 \\
6 & 3 & 2.5 & 3.1 & 2.8 & 1 & 2
\end{tabular}




\section{Al-Nahrain Journal of Science}

ANJS, Vol.23 (3), September, 2020, pp. $68-75$

Step 3: We make estimate of shape parameters $b, \beta_{1}, \beta_{2}, \beta_{3}$ of GIKD by (ranked set sampling and Bayes) methods as in (13), (14), (26), (27), respectively.

Step 4: We get estimation of Reliability as in: (15), (16), (28) and (29).

Step 5: We Calculate mean by: Mean $=\frac{\sum_{\mathrm{i}=1}^{\mathrm{L}} \widehat{\mathrm{R}}_{\mathrm{i}}}{\mathrm{L}}$
Step 6: Make comparison between the two different methods of estimation by using Mean Square Error:

$$
\operatorname{MSE}(\hat{R})=\frac{1}{L} \sum_{i=1}^{L}\left(\hat{R}_{i}-R\right)^{2}
$$

where $L$ represents number of replications for any experiment. The number of iterations is selected to be $L=500$, where $\hat{R}$ is the estimation of the $R$.

Simulation Results: Using the proposed estimation methods, the results presented in table (1) are obtained:

Table 1. The $\left(\hat{R}_{1}, \hat{R}_{2}\right.$ and MSE) values for experiment (1), such that $R_{1}-\mathrm{REAL}=0.666667, R_{2}-\mathrm{REAL}=0.733333$

\begin{tabular}{cccccccc}
\hline Sample size & $(\mathbf{1 5 , 1 5 , 1 5 , 1 5})$ & $\mathbf{( 3 0 , 3 0 , 3 0 , 3 0 )}$ & $\mathbf{( 9 0 , 9 0 , 9 0 , 9 0 )}$ & $\mathbf{( 1 5 , 3 0 , 3 0 , 9 0 )}$ & $\mathbf{( 3 0 , 9 0 , 9 0 , 3 0 )}$ & $(\mathbf{1 5 , 3 0 , 9 0 , 1 5 )}$ \\
\hline R1-RSS & 0.682477 & 0.662789 & 0.664249 & 0.460147 & 0.373669 & 0.361179 \\
MSE & 0.215099 & 0.005798 & 0.001192 & 0.061152 & 0.090361 & 0.110197 \\
R1BAYS & 0.668592 & 0.67122 & 0.668742 & 0.65865 & 0.662659 & 0.650191 \\
MSE & 0.004392 & 0.002439 & 0.000734 & 0.00425 & 0.00204 & 0.004687 \\
R2-RSS & 0.672071 & 0.748176 & 0.747907 & 0.492403 & 0.611991 & 0.622924 \\
MSE & 2.788304 & 0.004322 & 0.000952 & 0.07783 & 0.022828 & 0.549213 \\
R2BAYS & 0.751187 & 0.75265 & 0.751876 & 0.740252 & 0.748101 & 0.739332 \\
MSE & 0.003223 & 0.001969 & 0.000795 & 0.002915 & 0.001703 & 0.003379 \\
\hline
\end{tabular}

Table 2. The $\left(\hat{R}_{1}, \hat{R}_{2}\right.$ and MSE) values for experiment (2), such that $R_{1}-\mathrm{REAL}=0.666667, R_{2}-\mathrm{REAL}=0.733333$

\begin{tabular}{cccccccc}
\hline Sample size & $(\mathbf{1 5 , 1 5 , 1 5 , 1 5 )}$ & $\mathbf{( 3 0 , 3 0 , 3 0 , 3 0 )}$ & $\mathbf{( 9 0 , 9 0 , 9 0 , 9 0 )}$ & $\mathbf{( 1 5 , 3 0 , 3 0 , 9 0 )}$ & $\mathbf{( 3 0 , 9 0 , 9 0 , 3 0 )}$ & $\mathbf{( 1 5 , 3 0 , 9 0 , 1 5 )}$ \\
\hline R1-RSS & 0.69341 & 0.666679 & 0.668798 & 0.469369 & 0.379752 & 0.354138 \\
MSE & 0.955491 & 0.006958 & 0.001391 & 0.055282 & 0.086978 & 0.112599 \\
R1BAYS & 0.668336 & 0.665359 & 0.665222 & 0.654052 & 0.657681 & 0.657483 \\
MSE & 0.005085 & 0.002443 & 0.000894 & 0.003849 & 0.002277 & 0.003641 \\
R2-RSS & 0.678088 & 0.74925 & 0.751515 & 0.502276 & 0.618043 & 0.621868 \\
MSE & 4.289437 & 0.005443 & 0.001225 & 0.070349 & 0.01994 & 0.34652 \\
R2 BAYS & 0.749374 & 0.749026 & 0.748979 & 0.736536 & 0.743813 & 0.746596 \\
MSE & 0.00364 & 0.001857 & 0.000817 & 0.002498 & 0.001678 & 0.002859 \\
\hline
\end{tabular}

Table 3. The $\left(\hat{R}_{1}, \hat{R}_{2}\right.$ and MSE) values for experiment (3), such that $R_{1}-\mathrm{REAL}=0.666667, R_{2}-\mathrm{REAL}=0.733333$

\begin{tabular}{lcccccc}
\hline Sample size & $(\mathbf{1 5 , 1 5 , 1 5 , 1 5 )}$ & $\mathbf{( 3 0 , 3 0 , 3 0 , 3 0 )}$ & $\mathbf{( 9 0 , 9 0 , 9 0 , 9 0 )}$ & $\mathbf{( 1 5 , 3 0 , 3 0 , 9 0 )}$ & $(\mathbf{3 0 , 9 0 , 9 0 , 3 0 )}$ & $(\mathbf{1 5 , 3 0 , 9 0 , 1 5 )}$ \\
\hline R1-RSS & 0.677165 & 0.665116 & 0.669564 & 0.462873 & 0.382886 & 0.348717 \\
MSE & 0.208522 & 0.006668 & 0.00112 & 0.059008 & 0.084184 & 0.11974 \\
R1BAYS & 0.66922 & 0.669425 & 0.664688 & 0.657258 & 0.657042 & 0.656722 \\
MSE & 0.00442 & 0.002539 & 0.000731 & 0.003915 & 0.001963 & 0.004021 \\
R2-RSS & 0.772751 & 0.749515 & 0.752857 & 0.49588 & 0.622336 & 0.579007 \\
MSE & 0.254879 & 0.004658 & 0.001094 & 0.074584 & 0.01744 & 0.166795 \\
R2BAYS & 0.752265 & 0.752219 & 0.748124 & 0.73912 & 0.74346 & 0.744718 \\
MSE & 0.00305 & 0.001965 & 0.000683 & 0.00259 & 0.001445 & 0.003154 \\
\hline
\end{tabular}




\section{Al-Nahrain Journal of Science}

ANJS, Vol.23 (3), September, 2020, pp. $68-75$

\begin{tabular}{ccccccc}
\hline \multicolumn{6}{c}{ Table 4. The $\left(\hat{R}_{1}, \hat{R}_{2}\right.$ and MSE $)$ values for experiment $(4)$, such that $R_{1}-$ REAL $=0.651163, R_{2}-$ REAL $=0.736842$} \\
\hline Sample size & $(\mathbf{1 5 , 1 5 , 1 5 , 1 5 )}$ & $(\mathbf{3 0 , 3 0 , 3 0 , 3 0 )}$ & $\mathbf{( 9 0 , 9 0 , 9 0 , 9 0 )}$ & $(\mathbf{1 5 , 3 0 , 3 0 , 9 0 )}$ & $(\mathbf{3 0 , 9 0 , 9 0 , 3 0 )}$ & $(\mathbf{1 5 , 3 0 , 9 0 , 1 5 )}$ \\
\hline R1-RSS & 0.664226 & 0.666889 & 0.666586 & 0.474359 & 0.388022 & 0.377091 \\
MSE & 0.003605 & 0.001308 & 0.000523 & 0.034378 & 0.07008 & 0.078056 \\
R1BAYS & 0.669317 & 0.666299 & 0.667077 & 0.660967 & 0.660283 & 0.649599 \\
MSE & 0.005441 & 0.002512 & 0.000969 & 0.004262 & 0.001812 & 0.004275 \\
R2-RSS & 0.747617 & 0.749744 & 0.749766 & 0.510649 & 0.619613 & 0.613732 \\
MSE & 0.002452 & 0.000885 & 0.000358 & 0.05423 & 0.014887 & 0.019238 \\
R2 BAYS & 0.752593 & 0.750752 & 0.750419 & 0.742441 & 0.746379 & 0.739827 \\
MSE & 0.003532 & 0.001612 & 0.000665 & 0.002786 & 0.001436 & 0.003184 \\
\hline
\end{tabular}

Table 5. The $\left(\hat{R}_{1}, \hat{R}_{2}\right.$ and MSE) values for experiment (5), such that $R_{1}-\mathrm{REAL}=0.651163, R_{2}-\mathrm{REAL}=0.736842$

\begin{tabular}{ccccccc} 
Sample size & $(\mathbf{1 5 , 1 5 , 1 5 , 1 5 )}$ & $\mathbf{( 3 0 , 3 0 , 3 0 , 3 0 )}$ & $\mathbf{( 9 0 , 9 0 , 9 0 , 9 0 )}$ & $(\mathbf{1 5 , 3 0 , 3 0 , 9 0 )}$ & $(\mathbf{3 0 , 9 0 , 9 0 , 3 0 )}$ & $(\mathbf{1 5 , 3 0 , 9 0 , 1 5 )}$ \\
\hline R1-RSS & 0.665548 & 0.668007 & 0.666433 & 0.482746 & 0.385462 & 0.380036 \\
MSE & 0.00363 & 0.001387 & 0.000534 & 0.031316 & 0.071621 & 0.075891 \\
R1BAYS & 0.667748 & 0.665751 & 0.666357 & 0.652302 & 0.663003 & 0.650421 \\
MSE & 0.00514 & 0.002748 & 0.000981 & 0.004246 & 0.002113 & 0.0036 \\
R2 -RSS & 0.749777 & 0.750851 & 0.749564 & 0.518847 & 0.61718 & 0.619999 \\
MSE & 0.002507 & 0.000876 & 0.000356 & 0.050392 & 0.015644 & 0.017028 \\
R2 BAYS & 0.749839 & 0.749288 & 0.750107 & 0.735491 & 0.748952 & 0.738806 \\
MSE & 0.003311 & 0.00176 & 0.00066 & 0.002833 & 0.001566 & 0.002865 \\
\hline
\end{tabular}

Table 6. The $\left(\hat{R}_{1}, \hat{R}_{2}\right.$ and MSE) values for experiment (6), such that $R_{1}-\mathrm{REAL}=0.651163, R_{2}-\mathrm{REAL}=0.736842$

\begin{tabular}{cccccccc}
\hline Sample size & $(\mathbf{1 5 , 1 5 , 1 5 , 1 5 )}$ & $\mathbf{( 3 0 , 3 0 , 3 0 , 3 0 )}$ & $\mathbf{( 9 0 , 9 0 , 9 0 , 9 0 )}$ & $\mathbf{( 1 5 , 3 0 , 3 0 , 9 0 )}$ & $\mathbf{( 3 0 , 9 0 , 9 0 , 3 0 )}$ & $\mathbf{( 1 5 , 3 0 , 9 0 , 1 5 )}$ \\
\hline R1-RSS & 0.665742 & 0.664359 & 0.666531 & 0.47403 & 0.389742 & 0.377586 \\
MSE & 0.003469 & 0.001348 & 0.000571 & 0.03451 & 0.06929 & 0.077032 \\
R1BAYS & 0.670999 & 0.669295 & 0.666936 & 0.662551 & 0.657044 & 0.654584 \\
MSE & 0.005128 & 0.002657 & 0.001096 & 0.00423 & 0.001986 & 0.003456 \\
R2-RSS & 0.749104 & 0.747805 & 0.750195 & 0.510324 & 0.622943 & 0.61731 \\
MSE & 0.002348 & 0.000895 & 0.000386 & 0.054394 & 0.014069 & 0.01716 \\
R2 BAYS & 0.753297 & 0.752612 & 0.749792 & 0.743591 & 0.743186 & 0.74289 \\
MSE & 0.003446 & 0.001784 & 0.000689 & 0.002879 & 0.001368 & 0.00262 \\
\hline
\end{tabular}

\section{Conclusions}

The reliability, $R_{1}=P\left[Y<\max \left\{X_{1}, X_{2}\right\}\right]$, of two parallel components having $X_{1}, X_{2}$ Generalized Inverted Kumaraswamy strengths and exposed to $Y$ Generalized Inverted Kumaraswamy common stress with unknown shape parameter $\beta$ and common known shape $\alpha$ and scale $\gamma$ parameters is obtained. Then derived the reliability, $R_{2}=$ $P\left[Y<\max \left\{X_{1}, X_{2}, X_{3}\right\}\right]$, of three parallel components having $X_{1}, X_{2}, X_{3}$ Generalized Inverted Kumaraswamy strengths and exposed to $Y$ Generalized Inverted Kumaraswamy common stress.

MSE criteria used to make a comparison between two different estimators for each reliability function, where Bayes give the best performance for $R_{1}$ and $R_{2}$ after that ranked set sampling method is second order in the best.

I noticed and studied all experiments (tables) in simulation. We found $\hat{R}_{1}, \hat{R}_{2}$ in all sample sizes $\mathrm{A}, \mathrm{B}, \mathrm{C}, \mathrm{D}, \mathrm{E}, \mathrm{F}$ in experiments (Tables 1,2,3) that MSE is the least in Bayes method therefore Bayes method is first order in the best, again I found $\hat{R}_{1}, \hat{R}_{2}$ in sample sizes $\mathrm{D}, \mathrm{E}, \mathrm{F}$ in experiments 4, 5, 6 that MSE is the least in Bayes method therefore Bayes method is the best in the case while we found $\hat{R}_{1}, \hat{R}_{2}$ in sample sizes $\mathrm{A}, \mathrm{B}, \mathrm{C}$ in experiments 4, 5, 6 that MSE is the least in method ranked set sampling method therefore ranked set sampling method is the best in the case.

\section{References}

[1] Hassan A. S. and Basheikh H. M., (2012), "Estimation of Reliability in Multi-Component Stress-Strength Model Following Exponential Pareto Distribution", The Egyptian Statistical Journal, Institute of Statistical Studies Research, Cairo University, Vol. 56, No.2, pp. 82-95.

[2] Jonali Gogoi and Munindra Borah, "Interference on Reliability for Cascade Model", Journal of Informatics 


\section{Al-Nahrain Journal of Science}

ANJS, Vol.23 (3), September, 2020, pp. $68-75$

and Mathematical Sciences, Vol.4, No.1, pp 7783(2012).

[3] Sharma V.K.,Singh S.K, Singh U. and Agiwal V., (2014), "The Inverse Lindley Distribution: a StressStrength Reliability Model", Department of Statistics and DST-CIMS, Banaras Hindu, University Varanasi221005 , India.

[4] Hannagal D.D., (1998), "Estimation of system Reliability in stress - strength models For Distributions useful in life testing", IAPQR transactions, 23, 61 -65.

[5] Kumaraswamy, P. (1980) A Generalized Probability Density Function for Double- Bounded Random Processes. Journal of Hydrology, 46, 79-88.

[6] Abd Al-Fattah, A. M., El-Helbawy, A.A. and AlDayian, G.R. (2016), "Inverted Kumaraswamy Distribution: Properties and Estimation". Pakistan Journal of Statistics, 33, 37-61.

[7] Iqbal Z., Tahir M. M., A. Azeem S., Riaz N., Ahmad M., "Generalized Inverted Kumaraswamy Distribution: Properties and Application", Open Journal of Statistics, 2017, 7, 645-662.

[8] Khamnei H. J., Mayan S. R., (2016), "Comparison of Parameter Estimation in the Exponentiated Gumbel Distribution based on Ranked Set Sampling and Simple Random Sampling", Journal of Mathematics and Statistical Science, Vol.2016, 490-497.

[9] Stokes, L., (1995), "Parametric Ranked Set Sampling", Annals of the Institute of Statistical Mathematics, Vol. 47, No.3, pp. 465-482.

[10] Abbas N. S. and Maymona A. A., (2015), "Estimate the Shape Parameter of Generalize Rayleigh Distribution Using Bayesian - Shrinkage Technique", International Journal of Innovative Science, Engineering \& Technology, Vol. 2, Issue 6, ISSN $2348-7968$.

[11] Yakubu. A., Yahaya A., (2016), "Bayesian Estimation for the shape parameter of Generalized Rayleig1h distribution under non-informative Prior", International Journal of Advanced Statistics and Probability, Vol.4, No.1, pp.1-10. 